\title{
Variability Influence of Input Parameters on Result of Room Acoustic Calculation
}

\author{
D. MleczKo* AND T. WszołeK \\ AGH University of Science and Technology, Department of Mechanics and Vibroacoustics \\ Mickiewicza 30 Av., 30-059 Kraków, Poland
}

\begin{abstract}
For a long time there is a need in industry of acoustical modeling of rooms. Modeling is necessary for new production room design, the exchange of existing machinery, modernization or expansion of production rooms, changes in production profile or acoustical room adaptation for acoustical work conditions improvement. in such cases modeling quality is essential and thanks to uncertainty analysis it is possible to quantitatively estimate the effect that input parameters value variation has on model behavior. The article presents general rules for sound pressure level prediction uncertainty calculation in a room. By partial uncertainty calculation analysis of input parameters influence on uncertainty prediction an effort was taken to find parameters with biggest influence on the prediction process. As an example an industrial production room is presented which was modeled to predict noise level on a work stands after it was expanded.
\end{abstract}

PACS numbers: 43.20.Dk, 43.50.Jh, 43.55.Ka

\section{Introduction}

For a long time there is a need in industry of acoustical modeling of rooms. Modeling is necessary for new production room design, the exchange of existing machinery, modernization or expansion of production rooms, changes in production profile or acoustical room adaptation for acoustical work conditions improvement. In such cases modeling quality is essential and thanks to uncertainty analysis it is possible to quantitatively estimate the effect that input parameters value variation has on model behavior.

It is possible to prognose noise in industrial hall simplified, using ready models as well as computational algorithms. However, analysis of such causal state is appropriate from economic point of view. Acquaintance of influence of main noise sources and other factors on acoustic climate in room is necessary. Also it is important to define factors, in which investment is more effective from the point of view of correction of acoustic condition. A good quality (fidelity) of model is necessary to optimize costs. Estimate of it's uncertainty remains even after building and verification of given object. Further we want to know with what accuracy we have made a prognose and what is the influence of input data on it's result. Analysis of modeling uncertainty and fragmentary input parameters uncertainty is one of manner for model fidelity qualification.

\section{Uncertainty analysis}

Overall with modeling uncertainty estimation a sound pressure level distribution depends on a number of input

* corresponding author; e-mail: dmleczko@agh.edu.pl variables and each is loaded with a partial uncertainty which means that the result is a function of several variables [1,2]:

$$
L_{\mathrm{wy}}=f\left(X_{\mathrm{we}_{1}}, X_{\mathrm{we}_{2}}, \ldots, X_{\mathrm{we}_{k}}\right) .
$$

Then the total uncertainty could be calculated as:

$$
u_{c}\left(L_{\mathrm{wy}}\right)=\sqrt{\sum_{i=1}^{m}\left(\frac{\partial f}{\partial X_{\mathrm{we}_{i}}}\right)^{2} u^{2}\left(X_{\mathrm{we}_{i}}\right)},
$$

where: $u\left(X_{\mathrm{we}_{i}}\right)$ - standard partial uncertainties, related to all input variable uncertainties and effecting result of prediction.

The impact of partial uncertainty of parameters on the prediction uncertainty might be calculated by means of uncertainty propagation rule (1) for each parameter from the formula describing sound intensity at the receiver (4).

Sound intensity reaching the receiver by one path equals [3-5]:

$$
I_{i}=\frac{Q}{4 \pi d_{i}^{2}} \prod_{n=1}^{k}\left(1-\alpha_{n}\right) \exp \left(-m_{a} d_{i}\right)\left[\frac{W}{m^{2}}\right],
$$

where: $Q$ - power of sound, $d_{i}$ - length of a path number $i, \alpha_{n}$ - sound absorption coefficient of the surface reached as the $n^{\text {th }}$ one, $m_{a}$ - sound absorption coefficient of air.

Adding participations of all paths we will obtain the total sound intensity in the receiver:

$$
I_{c}=\sum_{i=1}^{l} I_{i}\left[\frac{W}{m^{2}}\right]
$$

where: $I_{i}$ - sound intensity reaching the receiver by the $i^{\text {th }}$ path $\left[\mathrm{W} / \mathrm{m}^{2}\right], l$ - number of paths.

After transformations we obtain the acoustic pressure level for the receiver $L_{c}$ : 
$L_{c}=L_{1}+10 \log W$,

where: $L_{1}=10 \log \frac{I_{1}}{I_{0}}$ - sound intensity level at a distance of $1 \mathrm{~m}$ from the source,

where: $I_{1}=\frac{Q}{4 \pi} \exp \left(-m_{a}\right)-$ sound intensity at a distance of $1 \mathrm{~m}$ from the source $\left[\mathrm{W} / \mathrm{m}^{2}\right], I_{0}-$ reference intensity $\left[\mathrm{W} / \mathrm{m}^{2}\right] . \quad W=\exp \left(m_{a}\right) \sum_{i=1}^{l} \prod_{n=1}^{k}(1-$ $\left.\alpha_{n}\right) \exp \left(-m_{a} d_{i}\right) d_{i}^{-2}-$ coefficient of sound amplification along paths.

Equation (4) is very convenient for the application since calculations are reduced solely to the determination of coefficient $W$. The level $L_{1}$ is known and given as the parameter describing the source.

Accordingly to above the following factors should be included formula to the parameters influencing the prediction result:

- related to the source:

- sound power of a source $Q$,

- directivity;

- related to the object:

- sound absorption coefficients $\alpha_{n}$ - there are many different surfaces in modeled room (e.g. case can stand at wall, put books on it etc.) and there is a question how precisely those coefficients should be imitated. How will uncertainty of whole model depend on each partial uncertainties (propagation of uncertainty),

- room model geometry accuracy,

- humidity, temperature and air pressure - related to sound absorption coefficient of air $m_{a}$ (uncertainties of those factors will be the same as measuring uncertainty)

- related to software - uncertainty introduced by software. As it was proven in [6], the same algorithm calculations, depending on the software package, which is implemented, can give significantly different results. Differing results may also be the result of different user assumptions such as: the computing grid density or the number of rays and reflections (ray tracing method) — it means qualification when increasing of these values practically does not produce changes in the calculation, but the time of calculation extends.

\section{Exemplary model}

Parallelepiped room was used for the preliminary calculations (Fig. 1).

A special half-omni-directional source, described in details in [7], will be used for acoustic tests. The room will serve as the basis for further calculations of the influence of input parameters on the results of acoustic calculations and their verification in real conditions.

\section{Results of calculations}

Based on above described model, the influence of individual input data change to acoustic pressure at the point of reception, has been calculated (Table).

TABLE

Change of largeness of measuring surface.

\begin{tabular}{l|c|c|c|c|c}
\hline \hline Surface $\left[\mathrm{m}^{2}\right]$ & 64.11 & 65.11 & 66.11 & 67.11 & 68.11 \\
\hline Deviation $\left[\mathrm{m}^{2}\right]$ & -2 & -1 & 0 & +1 & +2 \\
Result $[\mathrm{dB}]$ & 58.35 & 58.42 & 58.49 & 58.5 & 58.63 \\
Error $[\mathrm{dB}]$ & -0.14 & -0.07 & 0 & +0.01 & +0.14 \\
\hline \hline
\end{tabular}

Change of sound absorption coefficient.

\begin{tabular}{l|c|c|c|c|c}
\hline \hline Sound abs. coeff. & 0.1 & 0.2 & 0.3 & 0.4 & 0.5 \\
\hline Deviation & -0.2 & -0.1 & 0 & +0.1 & +0.2 \\
Result [dB] & 45.65 & 45.13 & 44.55 & 43.87 & 43.1 \\
Error [dB] & +1.1 & +0.58 & 0 & -0.68 & -1.45 \\
\hline \hline
\end{tabular}

Change of distance from source to receiver.

\begin{tabular}{l|c|c|c|c|c}
\hline \hline Distance [m] & 6.5 & 7.0 & 7.5 & 8.0 & 8.5 \\
\hline Deviation [m] & -1 & -0.5 & 0 & +0.5 & +1 \\
Result [dB] & 52.51 & 51.87 & 51.28 & 50.73 & 50.21 \\
Error [dB] & +1.23 & +0.59 & 0 & -0.55 & -1.07 \\
\hline \hline
\end{tabular}

Change of humidity.

\begin{tabular}{l|c|c|c|c|c}
\hline \hline Humidity [\%] & 30 & 40 & 50 & 60 & 70 \\
\hline Deviation [\%] & -20 & -10 & 0 & +10 & +20 \\
Result [dB] & 51.12 & 51.14 & 51.17 & 51.21 & 51.24 \\
Error [dB] & -0.05 & -0.03 & 0 & +0.04 & +0.07 \\
\hline \hline
\end{tabular}

Change of temperature.

\begin{tabular}{l|c|c|c|c|c}
\hline \hline Temperature $\left[{ }^{\circ} \mathrm{C}\right]$ & 15 & 17.5 & 20 & 22.5 & 25 \\
\hline Deviation $\left[{ }^{\circ} \mathrm{C}\right]$ & -5 & -2.5 & 0 & 2.5 & 5 \\
Result $[\mathrm{dB}]$ & 68.79 & 68.77 & 68.69 & 68.62 & 68.55 \\
Error $[\mathrm{dB}]$ & +0.1 & +0.08 & 0 & -0.07 & -0.14 \\
\hline \hline
\end{tabular}

Change of air pressure.

\begin{tabular}{l|c|c|c|c|c}
\hline \hline Air pressure $[\mathrm{hPa}]$ & 990 & 1000 & 1010 & 1020 & 1030 \\
\hline Deviation $[\mathrm{hPa}]$ & -20 & -10 & 0 & +10 & +20 \\
Result $[\mathrm{dB}]$ & 68.52 & 68.60 & 68.69 & 68.77 & 68.86 \\
Error $[\mathrm{dB}]$ & -0.17 & -0.09 & 0 & +0.08 & +0.17 \\
\hline \hline
\end{tabular}

Recapitulation.

\begin{tabular}{l|c|c|c|c|c}
\hline \hline Largeness changed & \multicolumn{5}{|c}{ Error [dB] } \\
\hline Distance & +1.23 & +0.59 & 0 & -0.55 & -1.07 \\
Sound abs. coeff. & +1.1 & +0.58 & 0 & -0.68 & -1.45 \\
Air pressure & -0.17 & -0.09 & 0 & +0.08 & +0.17 \\
Surface & -0.14 & -0.07 & 0 & +0.01 & +0.14 \\
Temperature & +0.1 & +0.08 & 0 & -0.07 & -0.14 \\
Humidity & -0.05 & -0.03 & 0 & +0.04 & +0.07
\end{tabular}




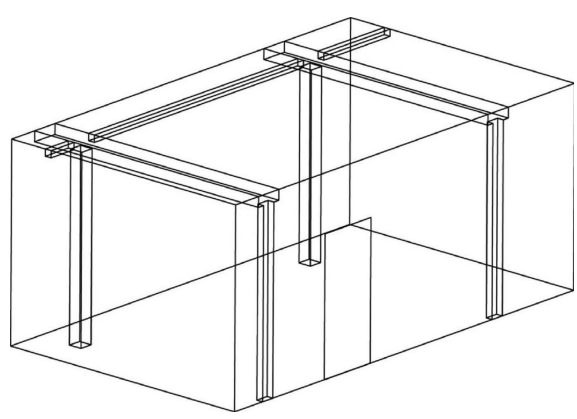

Fig. 1. Example of the cubical room.

\section{Conclusion}

In this article the foundation of algorithm assumptions of intensifying sound in closed area were presented. It helps to make sensitivity analysis of result account for acoustical model of room with input parameters changing. Using propagation of uncertainty partial uncertainties of those parameters influence for whole computations uncertainty where calculated.

Analysis of results of real object simulation account shows that the largest impact on the sound pressure level prediction result uncertainty in a room has a factor related to sound absorption coefficient of the surfaces reflecting the sound rays and distance form source to receiver accuracy. It was shown that all other parameters have a lower impact for model sensitivity.

The results that were obtained are in agreement with the ones that were expected. This confirms the correctness of the assumed model and approach and allows to hope that the detailed analysis of other results that would be obtained in various conditions will be also possible. This analysis will be conducted to other objects in subsequent work because in other areas this sensitivity may be different.

However all those results concern for particular accommodation, this approach can be employed in other objects also.

\section{References}

[1] Guide to the Expression of Uncertainty In Measurement, Int. Organization for Standardization (1993, 1995), p. 13.

[2] T. Wszołek, Uncertainty of sound insulation measurement in laboratory conditions - OSA, (2007) p. 192.

[3] A. Gołaś, Computer methods in room and enviromental acoustics, Wydawnictwa AGH, Kraków 1995, p. 190.

[4] J. Wierzbicki, in: Analysis of the room acoustic properties using simulation methods, Doctoral thesis, Kraków 1995, p. 21.

[5] H. Kuttruff, Room Acoustics, Applied Science Publishers Ltd, London 1979, p. 78.

[6] E. Hartog van Banda, H. Stapelfeldt, in: Implementing Prediction Standards in Calculation Software the Various Sources of Uncertainty, Int. INCE Symposium "Managing uncertainties in noise measurements and predictions: a new challenge for acousticians", Le Mans, France 2005.

[7] D. Mleczko, T. Wszołek, in: Characteristics of sound source using for acoustics measurements inside the room, 35rd Winter School on Vibroacoustical hazards suppression: Poland, Gliwice-Ustroń 2007, p. 111. 